\title{
Parallel implementation for HSLO(3)-FDTD with message passing interface on distributed memory architecture
}

\begin{abstract}
Finite-Difference Time-Domain (FDTD) method is a numerical method that can be used to solve electromagnetic problems in time domain. However, this method needs large computer memory and long execution time. Recently, a new scheme of FDTD has been develop which is called the High Speed Low Order (3) FDTD (HSLO(3)-FDTD) method which reduced the speed of FDTD method by $67 \%$. In this paper, we develop the parallel distributed version of the HSLO (3)-FDTD method on Distributed Memory Architecture Machine (Sunfire V1280) with message passing interface. We execute some numerical simulations by a new method with direct-domain (DD) approach to simulate one dimensional free space wave propagation represented by a Gaussian pulse. The simulation is conducted on 2 meter of solution domain truncated by a perfectly conducting boundary condition. The performance of the new schemes are analyzed and compared with the conventional parallel FDTD method in terms of processing time, speed-up, efficiency and cost. The new schemes is shown to be ultra-fast and very efficient with some superlinear speed-up.
\end{abstract}

Keyword: HSLO(3)-FDTD; FDTD; Parallel 